resulted in a worsening of pre-treatment serviceable hearing (OR of 0.26 , $\mathrm{p}<0.01$ ), in an increase of facial nerve impairment (OR of 1.62, p < 0.01), and showed a trend for increase trigeminal nerve impairment as well (OR of $1.42, \mathrm{p}=0.07)$. The incidence of vestibular symptoms and hydrocephalus requiring shunting could not be assessed since these measures were not reported consistently. CONCLUSIONS: The treatment of NF2-associated VS continues to pose a challenge, as current SRS regimens result in impaired hearing and worse cranial nerve comorbidities, despite achieving high tumor control. It remains unclear if these findings have to be intended as treatment complications or, rather, continuous disease progression despite tumor control.

NCOG-13. THE IMPACT OF CME ON THE KNOWLEDGE OF NEUROFIBROMATOSIS TYPE 1-RELATED TUMORS

Michelle Worst ${ }^{1}$, Kinjal Parikh ${ }^{2}$, Rich Caracio ${ }^{2}$, and Sara Fagerlie ${ }^{3}$; ${ }^{1}$ Medscape Oncology, Honolulu, HI, USA, ${ }^{2}$ Medscape Oncology, New York City, NY, USA, ${ }^{3}$ Medscape, New York City, NY, USA

BACKGROUND: Neurofibromatosis type 1 (NF1) is an autosomal dominant genetic condition characterized by a wide spectrum of clinical findings. Patients with NF1 develop benign and malignant tumors along the nerves of the skin, brain, and other body parts. Due to the unique presentation of each patient, clinicians are challenged to stay current with emerging data and how best to integrate new agents into multidisciplinary treatment paradigms. The objective of this study was to assess changes in neurologists' and pediatricians' knowledge and confidence through participation in education regarding NF1 treatment. METHODS: The educational activity consisted of a 30-minute online enduring, 1 faculty, chapterized summary of a live symposium. Educational effect was assessed with a repeated pairs pre-/post-assessment study with a 3-item, multiple choice, knowledge questionnaire and one confidence assessment question. For all questions, each participant served as his/her own control. Pre- and post-assessment scores were compared to determine relative changes in the proportion of correct responses. A chi-square test assessed statistical significance at the $P<0.05$ level. The activity launched $23^{\text {rd }}$ December 2019; data were collected until $2^{\text {nd }}$ April 2020. RESULTS: Overall, statistically significant improvements were seen after education for neurologists $(\mathrm{N}=171, P<0.05)$ and pediatricians $(\mathrm{N}=260, P<0.01)$. The relative improvement in correct answers was $26 \%$ for neurologists and $48 \%$ for pediatricians (percent of total correct responses pre-/post-assessment were $27 \% / 34 \%$ and $23 \% / 34 \%$, respectively). Following the activity, $16 \%$ of neurologists and $22 \%$ of pediatricians had a measurable increase in confidence regarding their understanding of multidisciplinary management of NF1. CONCLUSIONS: Participation in an online, 30-minute enduring CME intervention resulted in statistically significant improvements in knowledge and confidence of neurologists and pediatricians, that result in improvements in clinical care. Continued educational activities are paramount to address residual gaps and further increase clinicians' confidence in this clinical setting.

\section{NCOG-14. PSYCHOSOCIAL OUTCOMES IN CHILDREN WITH NEUROFIBROMATOSIS TYPE 1 AND PLEXIFORM NEUROFIBROMAS}

Diane Puccetti ${ }^{1}$, Paige Mission ${ }^{2}$, and Shawn Damodharan ${ }^{1} ;{ }^{1}$ American Family Children's Hospital, University of Wisconsin-Madison, Madison, WI, USA, ${ }^{2}$ University of Wisconsin-Madison, Department of Neurology, Madison, WI, USA

OBJECTIVE: This case series seeks to examine neurocognitive outcomes, social-emotional functioning, and family burden in young children diagnosed with Neurofibromatosis, type 1 (NF1) with early growing plexiform neurofibromas (PNFs). BACKGROUND: Neurofibromatosis, type 1 (NF1) is a common predisposing chronic disease arising in early childhood, with an incidence of approximately 1:3000. Though NF1 displays a wide range of phenotypic variability, the primary feature of the disease is peripheral nerve sheath tumors called neurofibromas. Less is well known regarding the broader neurocognitive and social-emotional profile in presentations with more complex tumor growths, namely PNFs, which are present in at least half of the NF1-affected population. METHODS: Participants with NF1 and PNFs $(n=2)$ aged 6-7years completed comprehensive neuropsychological evaluations and parents completed measures of quality of life, social-emotional/behavioral functioning of child, parental stress, family adaptability, and family cohesion. RESULTS: Outcomes suggest broad neurocognitive dysfunction (e.g., executive functioning deficits, attention problems, visual-motor delays, and poor motor coordination), socialemotional challenges (e.g., symptoms of anxiety and depression, and poor social skills), and familial distress. CONCLUSIONS: Findings indicate the value of early and frequent monitoring of children with PNFs in medical systems and multi-disciplinary teams, and the importance of early intervention for both children and families.

\section{NCOG-15. PREGNANCY AND GLIOMA}

Andrew Rodriguez, and Michael Ruff; Mayo Clinic, Rochester, MN, USA

Improvements in treatments and prognosis in glioma patients has led patients the opportunity for pregnancy despite their diagnosis. In this retrospective case series of 13 patients evaluated at Mayo Clinic, we identified patients who were pregnant at or after glioma diagnosis from 1998-2019. Clinical information was obtained through the electronic medical record Tumor grade, treatments, peripartum complications, and pediatric outcomes were analyzed. Median follow-up for pregnant patients was 13.5 years (range, 0-24). Of the 13 patients, 2 patients received temozolomide and radiation during pregnancy for glioblastoma and had no peripartum complications. Median survival time in these patients after further treatments was 23.5 months (range, 23.3-23.8). Two children from these pregnancies had no fetal malformations or pediatric developmental delays with median follow-up of 8.8 years (range, 4.7-12.8). Three patients received radiation during pregnancy for high grade gliomas and had no fetal malformations or peripartum complications. Eight patients had one or multiple pregnancies with median 2 (range, 1-3) children at or after diagnosis with 14 children total. Eleven of the 14 children had no fetal malformations or peripartum complications and were conceived without reproductive assistive technologies. Two children, who were identical twins, were born with a placental malformation but otherwise had normal pediatric development. One child was born pre-term and had long-term developmental delays.

NCOG-16. THE IMPACT OF THE TIMING OF HEALTH-RELATED QUALITY OF LIFE ASSESSMENTS ON THE ACTUAL RESULTS IN GLIOMA PATIENTS: A RANDOMIZED PROSPECTIVE STUDY

Marthe Peeters ${ }^{1}$, Hanneke Zwinkels ${ }^{2}$, Johan Koekkoek ${ }^{1}$, Maaike Vos ${ }^{2}$, Linda Dirven ${ }^{1}$, and Martin Taphoorn ${ }^{1}$; ${ }^{1}$ Leiden University Medical Center (LUMC), Leiden, Netherlands, ${ }^{2}$ Haaglanden Medisch Centrum (HMC), The Hague, Netherlands

BACKGROUND: The aim of this study was to explore the impact of the timing of health-related quality of life (HRQoL) measurements in clinical care on obtained HRQoL scores in glioma patients, and the association with feelings of anxiety or depression. METHODS: Patients completed the EORTC Quality of Life Questionnaires (QLQ-C30 and QLQ-BN20), and the Hospital Anxiety and Depression Scale (HADS) at two time points. All patients completed the first measurement on the day of the MRI-scan $(t=0)$, but the second measurement $(\mathrm{t}=1)$ depended on randomization; group 1 ( $\mathrm{n}=$ $49)$ completed the questionnaires before and group $2(n=51)$ after the consultation with the physician. RESULTS: Median HRQoL scale/item scores on $\mathrm{t} 0 / \mathrm{t} 1$ and change scores were comparable between the two groups. Between $8-58 \%$ of patients changed to a clinically relevant extent (i.e. $\geq 10$ points) on the evaluated HRQoL scales/items in about one week time, in both directions, with only $3 \%$ of patients remaining stable in all scales. The mean number of the 26 evaluated HRQoL scales/items that changed to a clinically relevant extent per patient was $7(S D=4)$. Patients with stable role functioning had a lower HADS anxiety change score. However, this association was no longer significant in the multivariable regression when corrected for confounding factors. The HADS depression score was not associated with a change in HRQoL. DISCUSSION: Measuring HRQoL before or after the consultation did not impact HRQoL scores on group level. However, the large majority of patients reported a clinically relevant difference in at least one HRQoL scale/item between the two time points, which was in general not influenced by feelings of anxiety or depression. Nevertheless, these findings highlight the importance of a standardized timing (e.g. at the day of the scan, consultation or first day of treatment) of patientreported outcome assessments in clinical trials.

\section{NCOG-17. PREDICTORS OF SURVIVAL IN ELDERLY PATIENTS UNDERGOING SURGERY FOR GBM}

Mathew Voisin, and Gelareh Zadeh; Princess Margaret Cancer Center, Toronto, ON, Canada

BACKGROUND: Despite the median age of diagnosis of GBM being 64 years old, there is only a paucity of studies on elderly patients with GBM. Furthermore, the majority of these studies examine treatment paradigms, and there is limited research on clinical and hospital factors on overall survival. The purpose of this study is to determine predictors of survival in elderly patients undergoing surgery for GBM. METHODS: We searched our hospital brain tumour biobank database for all consecutive patients over a 14-year period from 2005 to 2018 . All patients 65 years of age or older at time of surgery with a pathological diagnosis of de novo primary GBM were included. Kaplan-Meier survival curve and Cox proportional hazards model were constructed for overall survival vs age, sex, KPS, medical co-morbidities, extent of resection by surgeon, length of stay, postop complications, and discharge destination. RESULTS: A total of 150 patients were included. The median age at time of surgery was 74 years old (range: $65-94$ ). Median 
overall survival was 9.4 months (95\% CI: 7.8-12.2). Variables associated with worse survival included longer length of stay (HR: 1.15, $95 \%$ CI: $1.02-$ $1.30, \mathrm{p}=0.02$ ), discharge destination other than home (HR: 1.91, 95\% CI $1.01-3.6, \mathrm{p}=0.04$ ), and any postop complication (HR: $3.7,95 \%$ CI: 1.87 $7.3, p=<0.001)$. The presence of any or multiple medical comorbidities was not associated with worse survival $(\mathrm{p}=0.93$ and 0.19 , respectively). CONCLUSIONS: The presence of medical comorbidities is not associated with worse survival in elderly patients undergoing surgery for GBM. In order to maximize survival in these patients, avoidance of postoperative complications is paramount, along with a short hospital stay and attempt to discharge these patients to their home.

\section{NCOG-18. IS THE RANO CRITERIA FOR LOW-GRADE GLIOMA} RELIABLE IN THE CLINICAL SETTING? - A RELIABILITY STUDY

Erika Horta, Yueren Zhou, Laila M. Poisson, Brent Griffith, Michael Stone, James Snyder, and Tobias Walbert; Henry Ford Hospital, Detroit, MI, USA

INTRODUCTION: Magnetic resonance imaging (MRI) is a fundamental component of longitudinal neuro-oncology evaluation and decision-making. The Response Assessment in Neuro-Oncology criteria for low-grade glioma $\left(\mathrm{RANO}_{\mathrm{LGG}}\right)$ was designed as an outcome measure for clinical trials. Thus, this project intends to study the reliability of $\mathrm{RANO}_{\mathrm{LGG}}$ in the clinic setting. METHODS: 21 pairs of brain MRIs, that averaged three years apart, were selected from 21 patients with tissue diagnosis of WHO grade 2 gliomas. Two neuro-oncologists and two neuro-radiologists reviewed and independently scored the MRI pairs according to $\mathrm{RANO}_{\mathrm{LGG}}$ categories of progressive disease, stable disease, minor response, partial response, and complete response. Kappa-Fleiss (KF) was used to evaluate agreement among reviewers. RESULTS: Reviewers awarded identical scores in only $33 \%$ of MRI pairs and there was a complete disagreement in one MRI pair. Overall reliability of the criteria in the clinical setting is moderate $(\mathrm{KF}=0.44)$. Agreement between neuroradiologists $(\mathrm{KF}=0.51)$ and between neuro-oncologists $(\mathrm{KF}=0.48)$ were similar. Interpretation of post-contrast T1-weighted images had a better agreement $(\mathrm{KF}=0.31)$ than T2/FLAIRweighted images which had a poor agreement $(\mathrm{KF}=-0.02)$. Classification of progression versus non-progression had only a moderate agreement $(\mathrm{KF}=$ 0.49 ). History of radiation therapy or chemotherapy did not influence the criteria reliability (fisher exact text, $\mathrm{p}=0.58, \mathrm{p}=0.27$, respectively). CONCLUSION: $\mathrm{RANO}_{\mathrm{LGG}}$ reliability in the clinical setting is moderate, therefore it should be used cautiously for clinical decision-making. Other tools that can support the neuro-oncologist in the follow-up of patients with low-grade glioma are additional MRI sequences other than T2/FLAIR and contrast - weighted images, computer-aided diagnosis such as volumetrics, spectroscopy, positron emission tomography, and multidisciplinary tumor boards. Likewise, when image criteria for low-grade gliomas are designed, T2/FLAIR should be used guardedly, due to low interpretation agreement.

\section{NCOG-19. BEVACIZUMAB IN REAL LIFE PATIENTS WITH} RECURRENT GLIOBLASTOMA: BENEFIT OR FUTILITY?

Cristina Smolenschi ${ }^{1}$, Emeline Colomba ${ }^{2}$, Elie Rassy ${ }^{2}$, Naima Lezghed ${ }^{3}$, Mohamed Kettab ${ }^{2}$, Guillaume Louvel ${ }^{2}$, Gabriel Garcia ${ }^{3}$, Bianca Cheaib ${ }^{2}$, Sophie Bockel ${ }^{3}$, Johan Pallud ${ }^{4}$, Edouard Dezamis ${ }^{4}$, Razvan Copaciu ${ }^{5}$, Steven Knafo ${ }^{2}$, Samy Ammari ${ }^{2}$, Frederic Dhermain ${ }^{3}$, Julien Domont ${ }^{2}$, Larisa Martanovschi ${ }^{2}$, Fekih Mahmoud ${ }^{2}$, and Sarah Dumont ${ }^{3} ;{ }^{1}$ Gustave Roussy, France, ${ }^{2}$ Gustave Roussy, Paris, France, ${ }^{3}$ Institut Gustave Roussy, Villejuif, France, ${ }^{4}$ Centre Hospitalier Saint Anne, Paris, France, ${ }^{5}$ Hopital Bicetre, Paris, France

Angiogenesis represents a hallmark of glioblastoma but most trials disappointed and failed to change the poor outcome of this disease. However Bevacizumab (Bev) is widely used in clinical practice by expert oncologists due to experience or efficacy in real life. We retrospectively reviewed the use of Bev and its benefit in terms of Time to treatment failure (TTF), Overall Survival(OS), Objective Response Rate (ORR) and clinical benefit. METHODS: We analyzed two hundred and two patients treated at Gustave Roussy Cancer Campus with Bev until definitive failure for recurrent glioblastoma between 2006 and 2016. Patients were treated with Bev alone or in association with radiotherapy, temozolomide, lomustine or irinotecan. RESULTS: The median duration of Bev treatment until definitive failure was 6 months. The median TTF was 7.27 months $(95 \% \mathrm{CI} 6.30-8.24)$ and the median OS from diagnosis was 22.43 months( $95 \%$ CI 19.68-25.18). Two patients were still alive without active treatment at the end of study. A hundred and fourteen $(56 \%)$ patients experienced symptom amelioration and seventy-five $(37 \%)$ improved their Performance Status. Fifty percent of patients exhibited Partial and Complete Response on MRI, as best radiological response, within 1.6 months. No patient had anaphylactic reaction. Grade 1-2 hypertension(HT)(17\%) and grade $1(10 \%)$ proteinuria were most common. Six patients presented lethal toxicity: 4 with GI perforation, $1 \mathrm{p}$ with cerebral hemorrhage and $1 \mathrm{p}$ with arterial bleeding. HT was correlated with treatment response in $67 \%$ of patients. A neutrophil count superior to $6000 / \mathrm{mm}^{3}$ was associated with longer TTF(mTTF $8.23 \mathrm{~m}(95 \%$ CI 6.64
9.82). CONCLUSION: This retrospective study reports a substantial clinical benefit of Bev in patients with recurrent glioblastoma with an acceptable toxicity profile. As the panel of therapeutic option is still very limited in these tumors, this work supports the maintained use of Bev as a therapeutic option.

\section{NCOG-20. AWAKE SURGERY FOR RIGHT FRONTAL LOBE GLIOMA} CAN IMPROVE THE RESECTION RATE AND PRESERVE HIGHER COGNITIVE FUNCTIONS

Mitsutoshi Nakada $^{1}$, Riho Nakajima ${ }^{2}$, and Masashi Kinoshita ${ }^{1}$; ${ }^{1}$ Department of Neurosurgery, Kanazawa University, Kanazawa, Ishikawa, Japan, ${ }^{2}$ Department of Occupational Therapy, Kanazawa University, Kanazawa, Ishikawa, Japan

OBJECTIVE: Awake surgery is the standard treatment to preserve motor and language functions. The aim of this study is to evaluate the awake surgery for the patients with right frontal lobe (RFL) glioma on resection rate and preservation of higher cognitive functions. METHODS: Out of the 139 cases that underwent awake surgery at our hospital between 2013 and 2019 , 34 cases (mean age: 47.8 years) of RFL glioma were included in this study. The WHO classification was grade II, III, and IV for 15,13 , and 6 cases, respectively. We evaluated visual spatial cognition (VSC), spatial working memory (SWM), and social cognition (SC) before and after the surgery. These relevant areas were mapped intraoperatively. We did not map these areas in the cases in which the task could not be accomplished. Therefore each function was divided into an intraoperative evaluation group (EG) and a non-evaluation group (NEG), and the resection rate and functional outcomes were compared. RESULTS: The removal rate was significantly higher in the EG group for VSC and SC than that in the NEG ( $p=0.0078$ and 0.0024 , respectively). The chronic disability rate of VSC was significantly lower in the EG than that in the NEG $(5.6 \%$ vs. $31.3 \%, p=0.043)$. None of the patients had postoperative disability of SWM in the EG which is significantly lower than that in the NEG $(16.7 \%, \mathrm{p}=0.049)$. SC tended to have a lower disability rate $(20.0 \%)$ in the EG compared to that in the NEG $(45.8 \%)$. The probability that the posterior deep part of the middle frontal gyrus, which is the relevant area of VSC, was resected higher in the NEG ( $\mathrm{p}=$ $0.0052)$. CONCLUSIONS: We scientifically verified that the awake surgery for RFL glioma contributes to the improvement of resection rate and the preservation of higher cognitive functions.

\section{NCOG-21. INTERIM RESULTS OF THREE COGNITIVE REHABILITATION STRATEGIES IN PATIENTS WITH LOWER GRADE GLIOMAS}

Jennie Taylor ${ }^{1}$, Christina Weyer-Jamora ${ }^{2}$, Melissa Brie ${ }^{2}$, Paige Bracci ${ }^{3}$, Ellen Smith ${ }^{2}$, Tracy Luks ${ }^{2}$, Steve Braunstein ${ }^{4}$, Javier Villanueva-Meyer ${ }^{5}$, Karin Gehring ${ }^{6}$, Adrian Aguilera ${ }^{7}$, Nancy Ann Oberheim Bush ${ }^{5}$,

Jennifer Clarke ${ }^{1}$, Nicholas Butowski ${ }^{5}$, Susan $\mathrm{Chang}^{5}$, and

Shawn Hervey-Jumper ${ }^{5}{ }^{1}$ Department of Neurological Surgery, University of California (UCSF), San Francisco, San Francisco, CA, USA, ${ }^{2}$ University of California, San Francisco, San Francisco, CA, USA, ${ }^{3}$ Department of Epidemiology \& Biostatistics, UCSF, San Francisco, CA, USA, ${ }^{4}$ UCSF Medical Center, San Francisco, CA, USA, ${ }^{5}$ University of California San Francisco, San Francisco, CA, USA, ${ }^{6}$ Tilburg University, Tilburg, Netherlands, ${ }^{7}$ University of California, Berkeley, Berkeley, CA, USA

BACKGROUND: Patients with lower grade (2 and 3) gliomas (LrGG) are living longer, but often with cognitive impairments from their tumor and treatments. However, cognitive assessments and access to cognitive rehabilitation are not a standard part of care. We present preliminary results of a pilot study investigating feasibility and efficacy of three cognitive rehabilitation strategies for stable LrGG patients - in-person manualized cognitive rehabilitation; iPad based cognitive rehabilitation program of retraining and compensation strategies (ReMind); or daily instructional text messages (Healthy SMS). METHODS: Eligible patients were adults with clinically and radiologically stable LrGG, $>6$ months from last treatment, and $\geq 1$ standard deviation (SD) below normal on $\geq 2$ domains of neuropsychological assessments. Patients were first offered in-person cognitive rehabilitation or randomized to ReMind or Healthy SMS if unable to attend in-person. Interventions lasted 3 months. Neuropsychological and HRQOL assessments, using PROMIS NeuroQOL, were conducted at baseline, 3 , and 6 months post-intervention. Feasibility was defined as attending $\geq 80 \%$ of in-person sessions; completing $\geq 80 \%$ of ReMind tasks; or not opting out of Healthy SMS texts. RESULTS: To date 23/60 patients have enrolled: 11 in-person and 12 randomized to ReMind (5) or Healthy SMS (7). Demographic and clinical characteristics were similar between cohorts. Median age at testing was 46 years, with $65 \%$ female, and $78 \%$ having received prior radiation (median 4.1 years, range $3.2-11.5$ ). At baseline, processing speed was the most common domain of impairment with $43 \% \geq 1.5 \mathrm{SD}$ below normal and $36 \%$ patients reporting subjective cognitive impairment on HROQL assessment. Feasibility was $71 \%$ for in-person rehabilitation; $50 \%$ for ReMind; and $100 \%$ for Health SMS. CONCLUSION: These pre- 